\title{
Effective fluid transport properties of deformable rocks
}

Tobias M. Müller*, Geophysikalisches Institut, Universität Karlsruhe, Karlsruhe

Boris Gurevich, Curtin University and CSIRO Petroleum, Perth

\section{Summary}

Modern reservoir monitoring technologies often make use of diffusion waves in order to estimate the hydraulic conductivity and diffusivity of reservoir rocks. However, most theoretical descriptions for these effective fluid transport properties assume that the host rock is elastically rigid. Inhomogeneous poroelastic continua described by Biot's equations of dynamic or quasi-static poroelasticity provide an adequate framework to study the dependence of fluid transport properties on the elastic properties of the host rock. Analysis of diffusion wave fields in randomly inhomogeneous poroelastic structures provides new insight into how fluctuations of the compressible constituents of the rock affect the effective diffusivity. Using the method of statistical smoothing we derive an effective wave number of the coherent diffusion wave field. This wave number yields expressions for the effective conductivity and diffusivity of a deformable and inhomogeneous porous medium. These fluid transport properties are frequency-dependent. Comparison of the hydraulic conductivity derived here with that estimated from unsteady flow through porous media based on Darcy's law shows that they are identical in the limits of low and high frequencies.

\section{Introduction}

Diffusion waves are often used to characterize transport properties of fluid-saturated porous solids (Rice and Cleary, 1976). If the porous material has a deformable frame, the transport properties can be affected by the compressibility of the solid as well as fluid phase. This effect can be analyzed using Biot's theory of poroelasticity (Biot, 1962). In particular, Biot's theory predicts the existence of so-called Biot's slow wave, which in the lowfrequency (i.e., quasi-static) limit is governed by the diffusion equation (Chandler and Johnson, 1981). The corresponding diffusion coefficient depends on compressibilities of the fluid and solid phases as well as permeability and fluid viscosity.

Transport properties of porous materials are particularly affected by spatial heterogeneity. The study of these effects usually employs the concept of random media, and requires an analysis of field equations with random coefficients. One method that can be applied in this context is method of statistical smoothing (Karal and Keller, 1964), which has been widely used in the analysis of wave propagation in random media (Rytov et al., 1989 ) as well as flow through random rigid porous media (King, 1987; Keller, 2001).

In this paper we employ the method of statistical smoothing to compute the effective diffusivity of randomly inhomogeneous porous media. The medium is assumed to be governed by the low-frequency version of Biot's equations of poroelasticity where the slow compressional wave is a diffusion wave charac- terized by the wave number

$$
k_{0}=\sqrt{i \omega / D_{0}}
$$

where $\omega$ is angular frequency and $D_{0}$ the diffusivity of the porous medium (Norris, 1985). Neglecting the interaction with other wave modes, we analyze the coherent diffusion wave (slow wave) field only. We derive an expression for the effective diffusion wave number from which we extract the effective transport properties. We then discuss properties of these effective fluid transport parameters and compare our results to those obtained by similar approaches applied to fluid transport equations.

\section{Statistical smoothing in poroelastic media}

In random poroelastic media all parameters are represented by random fields of the form

$$
X=\bar{X}+\tilde{X}=\bar{X}\left(1+\varepsilon_{X}\right),
$$

where $\bar{X}$ is a constant background value and $\tilde{X}(\mathbf{r})$ is the fluctuating part. Parameter $\varepsilon_{X}=\tilde{X} / \bar{X}$ denotes the relative fluctuations and has zero mean $\left(\left\langle\varepsilon_{X}\right\rangle=0\right)$, autocorrelation function

$$
B_{X X}(\boldsymbol{\delta} \mathbf{r})=\left\langle\varepsilon_{X}(\mathbf{r}+\boldsymbol{\delta} \mathbf{r}) \varepsilon_{X}(\mathbf{r})\right\rangle
$$

and variance $\left\langle\varepsilon_{X}^{2}\right\rangle=B_{X X}(0)=\sigma_{X X}^{2}$.

The starting point of our analysis is the poroelastic Dyson integral equation for the mean Green's function of Biot's equations (Müller and Gurevich, 2005) in the random porous medium. By neglecting all contributions from the fast compressional and shear waves, we can write Dyson's equation for the matrix containing the mean Green's tensors $\overline{\mathbf{G}}$ in the form

$$
\overline{\mathbf{G}}=\mathbf{G}_{0}+\iint \mathbf{G}_{0} \mathbf{Q} \overline{\mathbf{G}},
$$

where $\mathbf{G}_{\mathbf{0}}$ denotes the matrix of Green's function for the homogeneous background (Müller and Gurevich, 2005)

$$
\mathbf{G}_{\mathbf{0}}=\frac{\kappa_{0}}{4 \pi i \omega} \partial_{i} \partial_{j} \frac{\exp \left(i k_{0} R\right)}{R}\left[\begin{array}{cc}
-\frac{C^{2}}{H^{2}} & \frac{C}{H} \\
\frac{C}{H} & -1
\end{array}\right],
$$

$R$ is the distance from source to observation point, $\partial_{i}$ denotes partial spatial derivative, $\mathbf{Q}$ is the matrix of the kernel-of-mass operators

$$
\mathbf{Q}=\left\langle\tilde{\mathbf{L}} \mathbf{G}_{0} \tilde{\mathbf{L}}+\int \tilde{\mathbf{L}} \mathbf{G}_{0} \tilde{\mathbf{L}} \mathbf{G}_{0} \tilde{\mathbf{L}}+\int \ldots\right\rangle,
$$

while $\tilde{\mathbf{L}}$ denotes the matrix of the perturbation operators

$$
\tilde{\mathbf{L}}=\left[\begin{array}{cc}
\partial_{i} \tilde{H} \partial_{j} & \partial_{i} \tilde{C} \partial_{j} \\
\partial_{i} \tilde{C} \partial_{j} & i \omega \tilde{p} \delta_{i j}+\partial_{i} \tilde{M} \partial_{j}
\end{array}\right]
$$




\section{Effective transport properties}

with the identity tensor $\delta_{i j}$. The method of statistical smoothing consists now in truncating $\mathbf{Q}$ after the first term. In Eqs. (5) and (6) $H$ is the undrained, low-frequency $P$-wave modulus given by Gassmann's equation

$$
H=P_{d}+\alpha^{2} M
$$

where

$$
M=\left[(\alpha-\phi) / K_{g}+\phi / K_{f}\right]^{-1}
$$

is the pore space modulus,

$$
P_{d}=K_{d}+4 / 3 \mu
$$

is the $P$-wave modulus of the drained frame,

$$
\alpha=1-K_{d} / K_{g}
$$

is the Biot-Willis coefficient, and $C=\alpha M$. Finally, $K_{g}, K_{d}$, and $K_{f}$ denote the bulk moduli of the solid phase, the drained frame, and the fluid phase resepctively, while $\mu$ denotes the porous-material shear modulus. In (5) the conductivity is denoted as $\kappa_{0}$ while in (6) $\tilde{p}$ denotes the fluctuating part of the reciprocal conductivity $p=1 / \kappa_{0}$. The diffusivity in (1) can be expressed through

$$
D_{0}=\kappa_{0} N
$$

where $N=M P_{d} / H$.

Eq. (4) contains a double spatial convolution which in the spatial Fourier domain yields a set of algebraic equations. Retaining only terms of order $O\left(\epsilon^{2}\right)$ yileds the following simplified equation for the $[2,2]$ component of $\overline{\mathbf{G}}$ :

$$
\bar{g}=g_{0}+\left(8 \pi^{3}\right)^{2} g_{0} q \bar{g},
$$

where $g_{0}$ and and $q$ are the spatial Fourier transforms of the corresponding components of $\mathbf{G}_{\mathbf{0}}$ and $\mathbf{Q}$, respectively. Assuming that the mean Green's function $\bar{g}$ is of the same functional form as $g_{0}$, but involving an effective wave number $k^{*}$, we can solve equation (12) for $k^{*}$. Evaluating the truncated kernel-of-mass operator matrix element $q$ for a statistically isotropic random medium we obtain the following approximation for the squared effective wave number

$$
k^{* 2}=k_{0}^{2}\left[1+\Delta_{s} \xi(\omega)\right],
$$

where

$$
\xi(\omega)=1+k_{0}^{2} \int_{0}^{\infty} r B(r) \exp \left(i k_{0} r\right) d r
$$

and

$$
\Delta_{s}=\left\langle\left(\frac{\alpha^{2} M}{P_{d}} \varepsilon_{\alpha}-\varepsilon_{K_{f}}+\varepsilon_{\phi}\right)^{2}\right\rangle+\frac{\sigma_{p p}^{2}}{3} .
$$

The method of statistical smoothing is precise for weak fluctuations only, and therefore, Eq. (13) is applicable if $\Delta_{s} \lesssim 1$. The physical interpretation of the effective diffusion wave number is as follows. Due to multiple scattering an initially homogeneous diffusion wave with the wavenumber $k_{0}$ becomes at finite frequencies an inhomogeneous diffusion wave characterized by $k^{*}$ (i.e., $\Re\left\{k^{*}\right\} \neq \Im\left\{k^{*}\right\}$ ). In the limits of zero and infinite

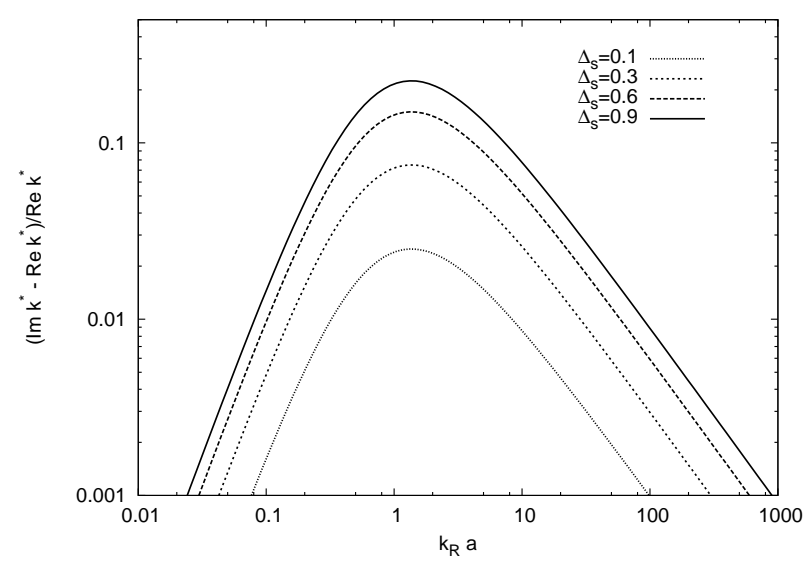

Fig. 1: Normalized difference of real and imaginary part of the effective wave number versus frequency for a model of a water-saturated, porous sandstone. For low and high frequencies this difference vanishes indicating the existence of a homogeneous diffusion wave. However, for finite frequencies the diffusion wave becomes inhomogeneous.

frequency the diffusion wave becomes homogeneous again with the effective wave numbers

$$
k^{*}(\omega \rightarrow 0)=k_{0}\left(1+\Delta_{s}\right)
$$

and

$$
k^{*}(\omega \rightarrow \infty)=k_{0},
$$

respectively. This behavior is also displayed in Figure 1.

\section{Effective hydraulic diffusivity and conductivity}

Effective wave number (13) can be used to construct approximation of the effective transport properties. Assuming that the effective wave number $k^{*}$ involves an effective diffusivity $D^{*}$ such that

$$
k^{*}=\sqrt{i \omega / D^{*}}
$$

we obtain

$$
D^{*}=D_{0}\left[1-\Delta_{s} \xi(\omega)\right] .
$$

The properties of this effective diffusivity are discussed next.

Figure 2 illustrates the frequency dependence of the effective diffusivity for varying $\Delta_{s}$ in a model of a porous sandstone where the inhomogeneities are statistically characterized by a correlation function of the form $B(r)=\exp (-|r| / a)$ where $a$ is the correlation length [shown is the real part of $D^{*}$ as a function of the dimensionless frequency $k_{R} a$ where $k_{R}$ denotes the real part of $k_{0}$ ]. As can be seen from Figure 2, the presence of inhomogeneities reduces the effective diffusivity relative to the background diffusivity. In the zero frequency limit we have

$$
D^{*}(\omega \rightarrow 0)=D_{0}\left(1-\Delta_{s}\right)
$$

whereas for infinitely high frequency the background value is obtained,

$$
D^{*}(\omega \rightarrow \infty)=D_{0} .
$$




\section{Effective transport properties}

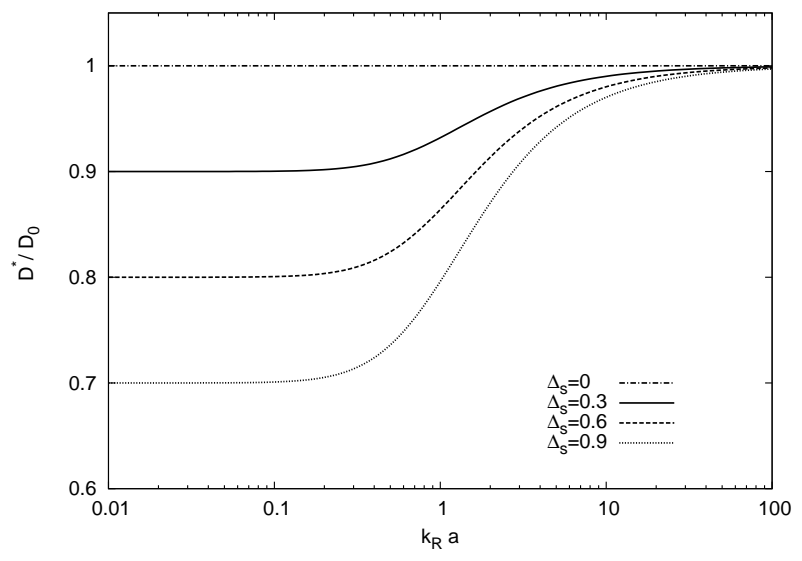

Fig. 2: Normalized effective diffusivity for varying $\Delta_{s}$ versus frequency for a porous sandstone model (shown is the real part of $D^{*}$ as a function of the dimensionless frequency $k_{R} a$ ). It can be observed that the presence of inhomogeneities reduces the effective diffusivity below the background diffusivity $D_{0}$.

The magnitude of the diffusivity dispersion is controlled by $\Delta_{s}$ which contains the second order moments of the random fields of $\alpha, \phi, K_{f}$ and $p$. The role of cross-correlations is particularly interesting. For example, negative cross-correlation between the Biot-Willis coefficient and the fluid bulk modulus, i.e. if there is a stiff fluid in the pore-space of a very compressible porous solid, produces an enhanced diffusivity dispersion.

Analogously to $D^{*}$, we can construct an effective conductivity $\kappa^{*}$ by assuming that the effctive wave number $k^{*}$ is of the form

$$
k^{*}=\sqrt{i \omega / \kappa^{*} N}
$$

i.e., $N$ is constant. In this case the calculations outlined above can be performed in all space dimensions and we obtain

$$
\kappa^{*}=\kappa_{0}\left[1-\frac{\sigma_{p p}^{2}}{m}\left(1-4 \int \frac{k_{R}^{4}}{4 k_{R}^{4}+K^{4}} \Phi(K) d \mathbf{K}\right)\right]
$$

in $m$-dimensional space $(\mathrm{m}=1,2$ or 3$)$ where $\Phi(K)$ denotes the fluctuation spectrum. This result is displayed in Figure 2. The effective conductivity is bounded by

$$
\kappa_{H} \leq \kappa^{*} \leq \kappa_{A}
$$

where $\kappa_{H}$ and $\kappa_{A}$ denote the harmonic and arithmetic average conductivities, respectively. In the low-frequency limit the lower bound becomes an identity for $m=1$ only, whereas at infinitely high frequencies the upper bound is reached exactly in all space dimensions.

\section{Similarity with results from analysis of unsteady flow}

For a poroelastic continuum the diffusion wave mode (Biot's slow wave) in the limit of zero frequency is equivalent to the quasi-static flow (Chandler and Johnson, 1981). It is therefore

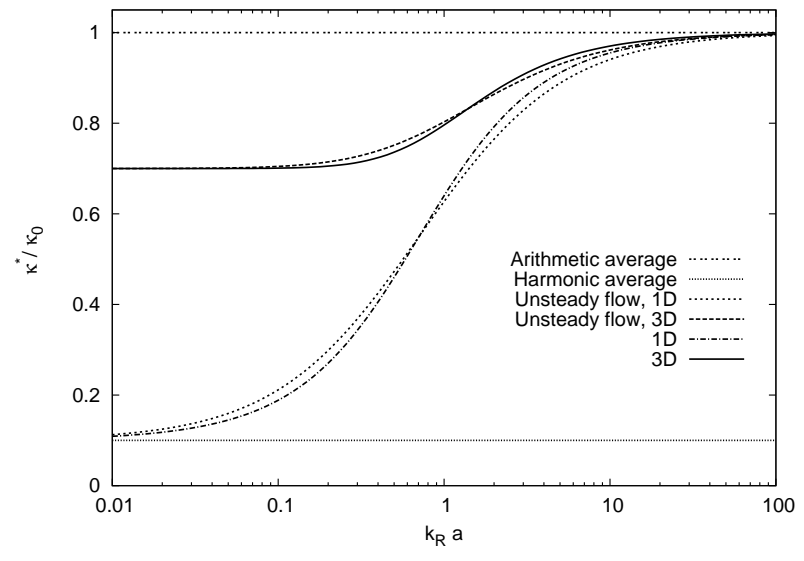

Fig. 3: Normalized effective conductivity based on approximations (23) and (26) versus dimensionless frequency in space dimension $\mathrm{D}=1$ and $D=3$. The exact bounds for the $1 \mathrm{D}$ case are also shown. The medium parameters are the same as in Figure 1 representing a porous sandstone model.

interesting to compare our results with estimates of an effective conductivity in random porous media based on Darcy's law (Keller, 2001). Time-dependent, i.e. unsteady, flow in a porous medium with a rigid frame analyzed on the basis of Darcy's law in conjunction with the time-dependent continuity equation yields a diffusion equation for the pore pressure of the form

$$
S \partial_{t} P=\nabla \cdot(\kappa \nabla P)
$$

where $S$ is the specific storativity (Dagan, 1982; Indelmann, 1996). In statistically isotropic $m$-dimensional random media an averaged Darcy law allows to define an effective conductivity. The latter can be explicitly computed for weakly inhomogeneous structures involving only the spatial correlation of the conductivity fluctuations (Indelmann, 1996):

$$
\kappa_{\text {flow }}^{*}=\kappa_{0}\left[1-\frac{\sigma_{\kappa \kappa}^{2}}{m} \int \frac{K^{2}}{k_{R}^{2}+K^{2}} \Phi(K) d \mathbf{K}\right] .
$$

This effective conductivity is also displayed in Figure 3. Note that the low and high frequency limits as well as the inflection point of $\kappa^{*}$ and $\kappa_{\text {flow }}^{*}$ [Eqs. (23) and (26)] are identical. Also, both approaches show that in the weak fluctuation case the effective conductivity does not depend on the compressibilities of the porous material and the fluid phase.

\section{Discussion and Conclusions}

The theoretical analysis of effective fluid transport properties has a long history in the fields of 'stochastic' hydrogeology and hydrogeophysics (e.g., Indelmann. 1996). In this paper we applied a relatively simple perturbation scheme to Biot's equations of dynamic poroelasticity in order to determine the effective fluid transport properties. We think that this concept is an attractive one since it opens the possibility to quantify the connection 


\section{Effective transport properties}

of fluid transport properties with 1.) general inhomogeneous porous materials where the diffusivity depends on the elastic properties of the surrounding rock material and with 2.) seismic wave propagation effects. In particular, the implications of these frequency-dependent fluid transport properties on seismic signatures is an interesting research topic.

We suspect that the difference between the solutions for the effective conductivity, Eqs. (23) and (26), is due to the different character of the governing partial differential equations. While Biot's equations of dynamic poroelasticity, used in this study, form a system of hyperbolic equations, the pore pressure diffusion equation is parabolic. We hypothesize that the use of quasistatic Biot's equations (e.g., Rice and Cleary, 1976), where the hyperbolicity is absent, would result in extactly the same effective conductivity.

In conclusion, the main result of this paper is the expression for the effective diffusivity (19). It is non-local and depends not only on the the second-order statistics of the conductivity but also on that of the poroelastic moduli. This results indicates that for accurate estimation of the effective conductivity from diffusion wave characteristics, fluctuations of the compressibilities of the porous material must be accounted for.

\section{Acknowledgments}

This work was kindly supported by the Deutsche Forschungsgemeinschaft (contract MU 1725/1-3).

\section{References}

Biot, M. A., 1962, Mechanics of deformation and acoustic propagation in porous media: J. Appl. Phys., 33, 1482-1498.

Chandler, R. N., and Johnson, D. L., 1981, The equivalence of quasistatic flow in fluid-saturated porous media and Biot's slow wave in the limit of zero frequency: J. Appl. Phys., 52, $3391-3395$

Dagan, G., 1982, Analysis of flow through heterogeneous random aquifers 2 . unsteady flow in confined formations: Water Resources Research, 18, 1571-1585.

Indelmann, P., 1996, Averaging of unsteady flows in heterogeneous media of stationary conductivity: J. Fluid Mech., 310, $39-60$.

Karal, F. C., and Keller, J. B., 1964, Elastic, electromagnetic and other waves in random media: J. Math. Phys., 5, 537-547.

Keller, J. B., 2001, Flow in random porous media: Transport in Porous Media, 43, 395-406.

King, P. R., 1987, The use of field theoretic methods for the study of flow in a heterogeneous porous medium: J. Phys. A: Math. Gen., 20, 3935-3947.

Müller, T. M., and Gurevich, B., 2005, A first-order statistical smoothing approximation for the coherent wave field in random porous media: J. Acoust. Soc. Am., 117, 4, 1796-1805.
Norris, A. N., 1985, Radiation from point source and scattering theory in a fluid-saturated porous solid: J. Acoust. Soc. Am., 77, 6, 2012-2023.

Rice, J. R., and Cleary, M. P., 1976, Some basic stress diffusion solutions for fluid-saturated elastic porous media with compressible constituents: Reviews of Geophysics and space physics, 14, 227-241.

Rytov, S. M., Kravtsov, Y. A., and Tatarskii, V. I., 1989, Wave propagation through random media:, volume 4 of Principles of statistical radiophysics Springer Verlag, Berlin. 


\section{EDITED REFERENCES}

Note: This reference list is a copy-edited version of the reference list submitted by the author. Reference lists for the 2006 SEG Technical Program Expanded Abstracts have been copy edited so that references provided with the online metadata for each paper will achieve a high degree of linking to cited sources that appear on the Web.

\section{REFERENCES}

Biot, M. A., 1962, Mechanics of deformation and acoustic propagation in porous media: Journal of Applied Physics, 33, 1482-1498.

Chandler, R. N., and D. L. Johnson, 1981, The equivalence of quasistatic flow in fluidsaturated porous media and Biot's slow wave in the limit of zero frequency: Journal of Applied Physics, 52, 3391-3395.

Dagan, G., 1982, Analysis of flow through heterogeneous random aquifers 2, Unsteady flow in confined formations: Water Resources Research, 18, 1571-1585.

Indelmann, P., 1996, Averaging of unsteady flows in heterogeneous media of stationary conductivity: Journal of Fluid Mechanics, 310, 39-60.

Karal, F. C., and J. B. Keller, 1964, Elastic, electromagnetic and other waves in random media: Journal of Mathematical Physics, 5, 537-547.

Keller, J. B., 2001, Flow in random porous media: Transport in Porous Media, 43, 395 406.

King, P. R., 1987, The use of field theoretic methods for the study of flow in a heterogeneous porous medium: Journal of Physics A: Mathematical and General, 20, 3935-3947.

Müller, T. M., and B. Gurevich, 2005, A first-order statistical smoothing approximation for the coherent wavefield in random porous media: Journal of the Acoustical Society of America, 117, 4, 1796-1805.

Norris, A. N., 1985, Radiation from point source and scattering theory in a fluid-saturated porous solid: Journal of the Acoustical Society of America, 77, 6, 2012-2023.

Rice, J. R., and M. P. Cleary, 1976, Some basic stress diffusion solutions for fluidsaturated elastic porous media with compressible constituents: Reviews of Geophysics and Space Physics, 14, 227-241.

Rytov, S. M., Y. A. Kravtsov, and V. I. Tatarskii, 1989, Wave propagation through random media, Principles of statistical radiophysics: Springer Verlag, 4. 